\title{
High excitation emission line nebula associated with an ultra-luminous X-ray source at $z=0.027$ in the AKARI North Ecliptic Pole Deep Field
}

\author{
J. Díaz Tello ${ }^{1,8}$, T. Miyaji ${ }^{1,8}$, T. Ishigaki ${ }^{2}$, M. Krumpe ${ }^{3}$, Y. Ueda ${ }^{4}$, H. Brunner ${ }^{5}$, T. Goto ${ }^{6}$, H. Hanami ${ }^{2}$, and Y. Toba ${ }^{7}$ \\ ${ }^{1}$ Instituto de Astronomía sede Ensenada, Universidad Nacional Autónoma de México, 22860 Ensenada, Mexico \\ e-mail: jadiazt@astrosen.unam.mx \\ 2 Physics Section, Faculty of Science and Engineering, Iwate University, 020-8551 Morioka, Japan \\ 3 Leibniz Institut für Astrophysik Potsdam, An der Sternwarte 16, 14482 Potsdam, Germany \\ ${ }^{4}$ Department of Astronomy, Kyoto University, 606-8502 Kyoto, Japan \\ 5 Max-Planck-Institut für extraterrestrische Physik, 8046 Garching, Germany \\ 6 National Tsinghua University, 30013 Hsingchu, Taiwan \\ 7 Academia Sinica Institute of Astronomy and Astrophysics, PO Box 23-141, 10617 Taipei, Taiwan \\ ${ }^{8}$ PO Box 439027, San Ysidro, CA 92143-9027, USA
}

Received 13 February 2017 / Accepted 2 June 2017

\begin{abstract}
Aims. We report our finding of a high excitation emission line nebula associated with an ultra-luminous X-ray source (ULX) at $z=0.027$, which we found in our Chandra observation of the AKARI North Ecliptic Pole Deep Field.

Methods. We present a Chandra X-ray and Gran Telescopio Canarias (GTC) optical spectral analysis of the ULX blob. We investigated the nature of the emission line nebula using line ratio diagnostic diagrams and estimated the physical properties of the nebula with spectral energy distribution (SED) fitting.

Results. The optical spectrum of this ULX blob shows emission line ratios that are located on the borderlines between star formation and Seyfert regimes in $[\mathrm{OIII}] / \mathrm{H} \beta-[\mathrm{OI}] / \mathrm{H} \alpha$, $[\mathrm{OIII}] / \mathrm{H} \beta-[\mathrm{SII}] / \mathrm{H} \alpha$, and $[\mathrm{OIII}] / \mathrm{H} \beta-[\mathrm{OIII}] /[\mathrm{OII}]$ diagnostic diagrams. These are in contrast with those of a nearby blob observed with the same slit, which occupies the HII regimes.

Conclusions. This result suggests that the energy input from the accretion power of the ULX, in addition to the star formation activity in the blob, significantly contribute to the ionization of the emission line nebula associated with the ULX, suggesting the existence of an accretion disk in the ULX emitting UV radiation or exerting shock waves.
\end{abstract}

Key words. X-rays: galaxies - X-rays: individuals: ANEPD-CXO104 - galaxies: individual: NEP J175536.7+664546.8

\section{Introduction}

Ultra-luminous X-ray sources (ULX) are off nucleus X-ray point sources found in galaxies that have $\mathrm{X}$-ray luminosities of roughly $\approx 10^{39}-10^{41} \mathrm{erg} \mathrm{s}^{-1}$, which are above the Eddington luminosity of a typical stellar mass black hole (Feng \& Soria 2011, for a recent review) and below those of a typical active galactic nucleus (AGN).

The most popular models of ULX involve either intermediate-mass black holes (IMBH) of $\approx 10^{2}-10^{4} M_{\odot}$ with standard accretion disks (Colbert \& Mushotzky 1999; Makishima et al. 2000) or stellar mass black holes $\left(\sim 10 M_{\odot}\right)$ accreting at super Eddington rates with isotropic emissions (Begelman 2002) and/or beamed emission within a small solid angle (e.g., King et al. 2001). These systems are frequently connected with massive star-forming regions, such as OB associations or superstar clusters, at the high-mass limit (Goad et al. 2002; Zezas et al. 2002; Liu et al. 2004).

Ultra-luminous X-ray sources are often located inside nebulae. Optical spectral information on ULXs and the surrounding nebula is still scarce and mostly limited to nearby ( $\$ 10$ Mpc) ULXs (Pakull \& Mirioni 2002; Abolmasov et al. 2007; Gutiérrez \& Moon 2014; Fabrika et al. 2015). A common notable feature is the HeII $\lambda 4686$ emission line, along with other common emission lines, such as $[\mathrm{OI}] \lambda 6300$, indicating a high excitation UV radiation nebula and/or shocks (e.g., Kaaretl et al. 2004). Integral field spectroscopy around the ULX Holmberg II $\mathrm{X}-1$ shows that, while the HeII emission is concentrated at the ULX position, there is an extended nebulosity of emission lines, including Balmer, [OIII], and [SII] lines extending toward one side of the ULX (Lehmann et al. 2005; Fabrika et al. 2015) that probably trace an ionization cone. From emission line diagnostics of nearby ULX nebulas, Abolmasov et al. (2007) argued that some of these sources are photoionized, while others are shock ionized. In addition, Feng \& Soria (2011) summarized information about X-ray photoionized and shock-ionized nebulae.

As part of our Chandra survey in the AKARI North Ecliptic Pole (NEP) Deep Field $(\mathrm{RA}=17: 55: 24$, dec $=+66: 37: 23$, Krumpe et al. 2015; Matsuhara et al. 2006), we found a new ULX in the star-forming galaxy NEP J175536.7+664546.8 at $z=0.027$ (Shim et al. 2013; $D \approx 110 h_{70}^{-1} \mathrm{Mpc}$ ). In this article, we present X-ray and optical spectral analyses of the ULX and an associated nebula and star cluster. In Sect. 2 we describe the basic X-ray properties of the ULX and show results of our $\mathrm{X}$-ray spectral analysis; in Sect. 3 the optical spectrum is presented; while in Sect. 4 we analyze the nature of the emission 
Table 1. X-ray observations ( $1 \sigma$ errors).

\begin{tabular}{ccccccc}
\hline \hline OBSID & $\begin{array}{c}\text { Date obs. } \\
{[\text { Y-M-D] }}\end{array}$ & $\begin{array}{c}\text { Exp. time } \\
{[\mathrm{ks}]}\end{array}$ & $\begin{array}{c}\text { CR }(0.5-7 \mathrm{keV}) \\
{\left[10^{-4} \mathrm{cts} \mathrm{s}^{-1}\right]}\end{array}$ & $\begin{array}{c}\text { Offax. } \\
{[\operatorname{arcmin}]}\end{array}$ & $\begin{array}{c}\text { Src. area } \\
{\left[\operatorname{arcsec}^{2}\right]}\end{array}$ & $\begin{array}{c}\text { Bkg. area } \\
{\left[10^{4} \operatorname{arcsec}^{2}\right]}\end{array}$ \\
\hline 12929 & $2011-03-16$ & 11.9 & $7.2 \pm 2.5$ & 2.6 & 28.3 & 2.2 \\
12930 & $2011-04-01$ & 14.6 & $9.7 \pm 2.7$ & 6.7 & 79.2 & 7.5 \\
12933 & $2010-12-28$ & 23.8 & $10.0 \pm 2.0$ & 6.5 & 200.9 & 4.3 \\
13244 & $2011-04-02$ & 14.9 & $9.4 \pm 2.7$ & 7.3 & 199.0 & 2.2 \\
\hline
\end{tabular}

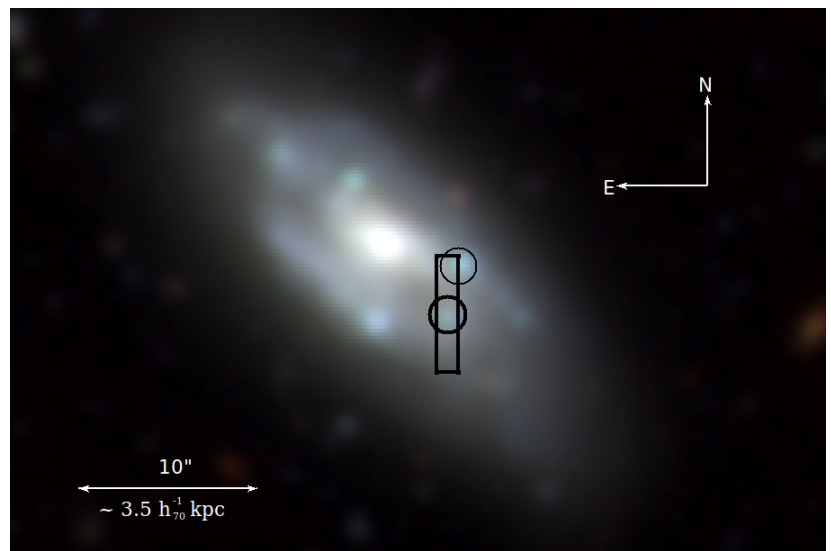

Fig. 1. Composite image from BRz Subaru Suprime Cam images of the galaxy NEP J175536.7+664546.8. The thick and thin circles show the positions of the ULX and nearby blobs, respectively, while the size of the circles corresponds to the aperture photometry used for the SED modeling. The rectangle approximately shows the slit geometry utilized in the OSIRIS/GTC spectroscopy.

line nebula and star cluster associated with the ULX and discuss its physical properties. Finally, Sect. 5 discusses the implications of our results and Sect. 6 summarizes our conclusions. Throughout this article we have assumed a flat cosmology with $\Omega_{\mathrm{m}}=0.3$, $\Omega_{\Lambda}=0.7$ and $H_{0}=70 h_{70} \mathrm{~km} \mathrm{~s}^{-1} \mathrm{Mpc}^{-1}$.

\section{X-ray properties}

The Chandra X-ray source ANEPD-CXO104 (CID = 104 in Krumpe et al. 2015) is located at RA $=17: 55: 36.28$, Dec $=$ $66: 45: 43.8$ (J2000) with a $1 \sigma$ positional error of $\approx 0.25^{\prime \prime}$ including both statistical and systematic errors. The X-ray source is co-spatial with one of several seemingly HII-region knots along spiral structures of NEP J175536.7+664546.8 and is 5.6" (projected distance of $\sim 3.1 \mathrm{kpc}$ ) away from the center of the galaxy. Figure 1 shows an optical image of the galaxy with the position of the ULX indicated with a thick circle.

The X-ray source has $\sim 80$ net counts and we only perform a minimal spectral analysis. For each observation identifier (OBSID), the ULX spectra are extracted from a circle centered at the source position. The size of the circle depends on the off-axis angle, source brightness, and background level. To minimize fluctuations of the background level, background spectra are extracted from a large irregular-shaped polygonal area on the same chip as the ULX. We have taken care to make sure no bright sources and visible structures are included in the background area. The mean off-axis angle of the area is close to that of the ULX and the background surface brightness of various subsections are consistent with that of the background inmediately surrounding the source. The source and background extraction areas are listed in Table 1. Then, we carried out the spectral analysis with a joint fit to four X-ray spectra of

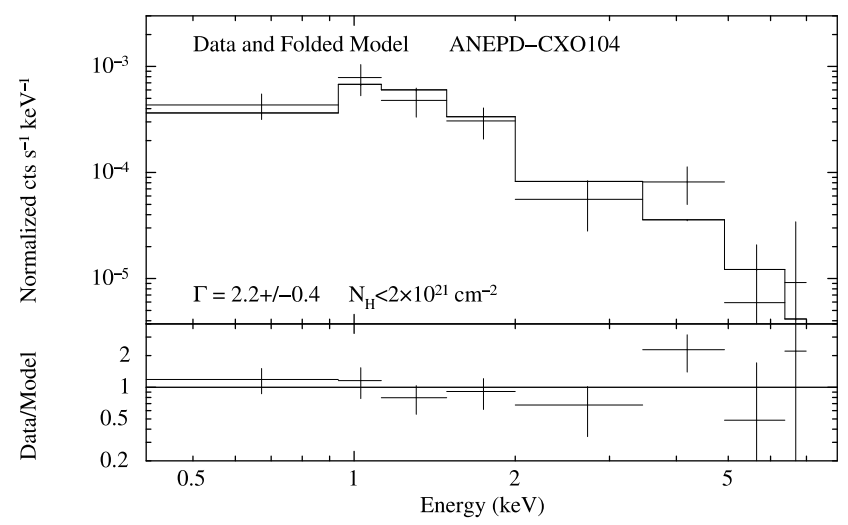

Fig. 2. X-ray spectrum of ANEPD-CXO104 folded with the instrumental response. The upper section shows the folded spectrum with error bars and with our best fit model. The residuals of the fit are shown in terms of data/model ratio (lower section). The error bars on data points correspond to $1 \sigma$ errors, while the error shown for $\Gamma$ is for $90 \%$ confidence level.

ANEPD-CXO104 accumulated from four Chandra observations with a simple absorbed power-law model of the photon spectrum; i.e., $P(E) \propto \operatorname{phabs}\left(N_{\mathrm{H}}^{\mathrm{Gal}}\right) \cdot \operatorname{zphabs}\left(z, N_{\mathrm{H}}^{\mathrm{src}}\right) \cdot E^{-\Gamma}$, where phabs ${ }^{1}$ represents a photoelectric absorption in our galaxy with the column density of $N_{\mathrm{H}}^{\mathrm{Gal}}=4 \times 10^{20} \mathrm{~cm}^{-2}$ (fixed to the Galactic value at the position of the source; Kalberla et al. 2005), $\operatorname{zphabs}\left(z, N_{\mathrm{H}}^{\mathrm{src}}\right)$ is the photoelectric absorption at the redshift of the source $(z=0.027)$, and $\Gamma$ is the photon index of the primary continuum of the source. The free parameters $N_{\mathrm{H}}^{\mathrm{src}}$ and $\Gamma$ for the all four spectra were limited, while the normalizations were allowed to vary individually. The fit was made using the "cstat" statistic in the XSPEC ${ }^{2}$ package, which fully incorporates Poisson errors of the pulse-height spectra of the source and background. As a result, the slope of the primary continuum is found to be $\Gamma=2.2 \pm 0.4$ (90\% confidence errors). No evidence for intrinsic absorption is found with a $90 \%$ upper limit of $N_{\mathrm{H}}<$ $2 \times 10^{21} \mathrm{~cm}^{-2}$. Also, there is no significant variability among the four observations. To obtain a representative X-ray flux, we then joined the normalizations and refit. The best fit $\Gamma$ and the upper limit of $N_{\mathrm{H}}^{\mathrm{src}}$ changed very little after joining the normalization. We find $S_{0.5-7 \mathrm{keV}}=(1.4 \pm 0.3) \times 10^{-14} \mathrm{erg} \mathrm{s}^{-1} \mathrm{~cm}^{-2}(0.5-7 \mathrm{keV}$, before Galactic absorption), implying an intrinsic X-ray luminosity of $L_{0.5-7 \mathrm{keV}} \approx 2.4 \pm 0.5 \times 10^{40} h_{70}^{-2} \mathrm{erg} \mathrm{s}^{-1}$ (90\% confidence errors). Thus, this source can be classified as a ULX. Figure 2 shows the X-ray spectrum folded with the instrumental response, our best fit model and fit residuals. The summed spectrum from all four OBSIDs (rather than separate four spectra) was rebinned, for display purposes only, to have at least $3 \sigma$ signals per bin of the source spectrum before the background

\footnotetext{
1 More details can be found in Xspec package manual.

2 Available at

https://heasarc.gsfc.nasa.gov/xanadu/xspec/
} 

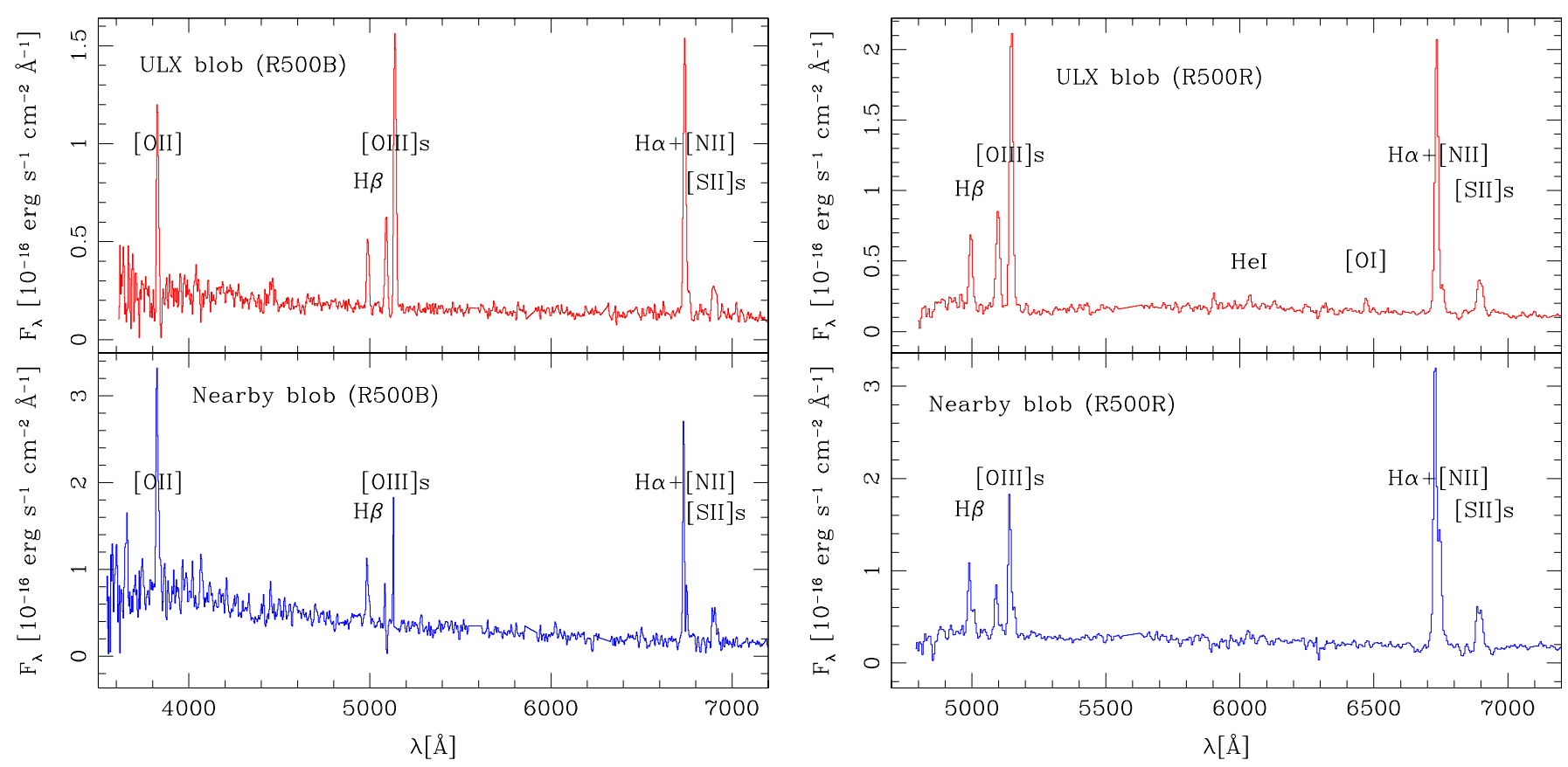

Fig. 3. One-dimensional spectra (observed frame) of the ULX ANEPD-CXO104 (upper sections) and nearby blob (lower sections) obtained with OSIRIS/GTC. The left panel shows the spectra obtained with the R500B grism, while the right panel shows the spectra obtained with the R500R grism. Strong residual sky emission lines were removed from the spectra.

subtraction. The plotted model uses the best fit parameters of the joint fit with the joined normalization.

\section{Optical spectroscopy}

Optical spectra were obtained for the nebula associated with our ULX ANEPD-CXO104 (hereafter referred as the "ULX blob") with the Optical System for Imaging and low-intermediateResolution Integrated Spectroscopy (OSIRIS) of the Gran Telescopio Canarias (GTC), as part of a spectroscopic survey of optical counterparts of Chandra X-ray sources detected in the AKARI NEP Deep Field. Multi-object spectroscopy was performed in service mode (PI: T. Miyaji) on the nights of April 23 and 26 of 2015 with the R500B and R500R grisms, which gave a dispersion of 3.54 and $4.88 \AA$ /pix, respectively. The R500B grism was used during the first night (Prod ID = 778892-778894), while the R500R grism was used during the second night (Prod ID = 779571-779573). With these grisms, the nominal spectral coverages are from 360 to $700 \mathrm{~nm}$ for R500B and from 500 to $1000 \mathrm{~nm}$ for R500R, respectively. However, in some cases, the R500B grism practically provided good spectra from 360 to $1000 \mathrm{~nm}$, but with a lower sensitivity toward redder wavelengths. It was useful to have two measures of the features of an object. The slit width adopted during the observations was $1.2^{\prime \prime}$ (geometry drawn in Fig. 1), which is a value higher than the average seeing of the whole run ( $\left.1^{\prime \prime}\right)$. Integration times of $3 \times 690 \mathrm{~s}$ and $3 \times 1100 \mathrm{~s}$ were used for R500B and R500R grisms, respectively, to reach a signal-to-noise ratio over 3 in the spectra obtained during the observations. Usual calibration frames were acquired during the run plus standard star spectra to calibrate with flux.

The data reduction process was carried out via the gtcmos ${ }^{3}$ package, which works in IRAF. Before using this package, the

\footnotetext{
3 Developed by Divakara Mayya at INAOE, Puebla, Mexico.
} Available at www . inaoep . mex/ ydm/gtcmos/gtcmos.html
OSIRIS images composed of two CCDs with a gap and a slight shift and rotation between them, were combined into a single mosaic frame with their pixel coordinates geometrically corrected. This process was performed via the mosaic $2 x 2 \_v 2$, task available on the GTC website. Then, the images were combined and bias subtracted using standard routines of IRAF. The arc line identification, wavelength calibration, and sky subtraction steps were carried out with the omidentify, omreduce, and omskysub routines, respectively, to obtain the calibrated two-dimensional spectra. The one-dimensional spectra were extracted via the omextract routine, while the standard stars were extracted with the apall routine. Finally, the flux calibration process was performed with the standard, sensfunction, and calibrate routines, also within IRAF.

Along with a spectrum of the ULX blob, the spectrum of another nearby blob (hereafter referred as the nearby blob) was also partially taken within the same slit. These objects resulted in having the same redshift of the host galaxy $(z=0.027)$, which indicates that both objects indeed belong to the galaxy. Figure 3 shows the spectra of both objects obtained with the R500B (left panel) and R500R (right panel) gratings. We can see both sources are active regions with strong Balmer, [OII] $\lambda 3729$, and [OIII] 24959,5007 emission lines. A quick look indicates that our ULX ANEPD-CXO104 has a spectrum resembling a Seyfert 2, while the nearby blob has a spectrum resembling a star-forming region. The HeI $\lambda 5876$ line and [OI] $\lambda 6300$ line in emission in the R500R spectrum of the ULX blob is also seen. The HeII $\lambda 4686$ emission line, which is commonly observed in optical spectra of and around ULX (Kuntz et al. 2005), has not been detected in our spectra.

Based on the FWHMs of narrow night sky lines, the spectral resolution achieved is roughly $\lambda / \delta \lambda_{\mathrm{FWHM}}=463$ and 440 for the R500B (7000 $\AA$ ) and R500R (7000 $)$ ), respectively. For the nearby blob, a somewhat higher spectral resolution was achieved because the nearby blob only overlaps partially with the slit. 
Table 2. Physical properties.

\begin{tabular}{ccccccc}
\hline \hline Metallicity & Object & $\tau[\mathrm{Gyr}]$ & Age[Gyr $]$ & $E(B-V)$ & $M_{*}\left[M_{\odot}\right]$ & $S F R\left[M_{\odot} / \mathrm{yr}\right]$ \\
\hline$Z_{\odot}$ & ULX blob & 15 & $2.00 \pm 1.22$ & 0.1 & $(3.63 \pm 1.13) \times 10^{7}$ & $0.022 \pm 0.008$ \\
& Nearby blob & $\infty$ & $1.43 \pm 0.45$ & 0 & $(2.39 \pm 0.30) \times 10^{7}$ & $0.021 \pm 0.003$ \\
\hline $0.4 Z_{\odot}$ & ULX blob & 30 & $0.09 \pm 0.07$ & 0.4 & $(1.29 \pm 0.51) \times 10^{7}$ & $0.162 \pm 0.096$ \\
& Nearby blob & $\infty$ & $0.18 \pm 0.15$ & 0.2 & $(1.20 \pm 0.54) \times 10^{7}$ & $0.078 \pm 0.055$ \\
\hline
\end{tabular}

\section{Diagnostic of the blobs}

In this section, we study the properties of the ULX blob by analyzing optical to near-infrared continuum and optical emission lines. These emission lines are compared with those of the nearby star-forming blob for reference.

From the image (Fig. 1), it appears that both the ULX and nearby blobs are associated with a star-forming region/star cluster. In order to investigate the environment in which the ULX was formed, we first studied the stellar populations of these blobs. The photometric data were extracted from broadband images obtained with CFHT Mega Cam $\left(u^{*}\right)$, Subaru Suprime Cam $\left(B, V, R_{\mathrm{c}}, i^{\prime}, z^{\prime}\right)$, and CFHT WIRCAM $\left(Y, J, K_{\mathrm{s}}\right)$ cameras on the AKARI NEP Deep Field (Takagi et al. 2007; Hanami et al. 2012; Murata et al. 2013; Oi et al. 2014). A $2^{\prime \prime}$ aperture was used for the photometry, which is shown as a circle in Fig. 1. The magnitude error associated with the aperture photometry was $\sim 0.02$ mag. We subtracted the contributions from emission lines detected in our GTC spectra from the photometric data, assuming that the rest of broad line fluxes are dominated by continuum emission from stars. In our particular case, only the photometry from $u^{*}, V$, and $R$ filters needed a correction, where $\sim 0.4 \mathrm{mag}$ is the largest correction for $R$ filter and $\sim 0.2 \mathrm{mag}$ is the smallest correction for $u^{*}$. We then applied spectral energy distribution (SED) fittings using stellar population synthesis models. We generated SED templates via the GALAXEV code (Bruzual \& Charlot 2003) with ages of [0, 13] Gyr and two values of metallicity, $Z_{\odot}$ and $0.4 Z_{\odot}$, respectively. We assumed a Salpeter initial mass function (IMF; Salpeter 1955) and exponentially declining star formation histories $\left(S F R(t) \sim \exp ^{-t / \tau}\right)$ with $\tau$ values of $[0, \infty]$ Gyr. A reddening law (Allen 1976) was applied to each template with $E(B-V)$ values of $[0,1]$. Then, we used the photometric redshift code Hyperz (Bolzonella et al. 2000) for the SED fitting. Table 2 shows the properties estimated for each object, while Fig. 4 shows the emission line subtracted photometries and the best fit models for each blob. The results of the SED fits show similar star formation histories between the ULX and nearby blobs at both assumed metallicities. These metallicities both show almost constant star formation rates (SFR) with comparable ages, which are consistent with each other within errors in either choice of the metallicity. If we assume a subsolar metallicity, the SED fits imply higher current SFR, which started more recently than the solar metallicity case. In any case, the similarity suggests that both star-forming blobs were born simultaneously some $0.1-2$ Gyr ago, depending on the assumed metallicity. Nevertheless, the subsolar metallicity case is likely to be correct as it is discussed in Sect. 5 .

We then analyzed the emission line properties of the blobs observed by GTC. The flux scales of the GTC spectra were normalized to $2^{\prime \prime}$ aperture fluxes with the $B-, V-, R_{\mathrm{c}}-$, $i$-photometric measurements from the images described above. This procedure compensates for flux losses caused by the small slit width of the spectroscopic observations. Then the contributions from stellar population continua, which contain features such as Balmer absorption, were subtracted from the spectra

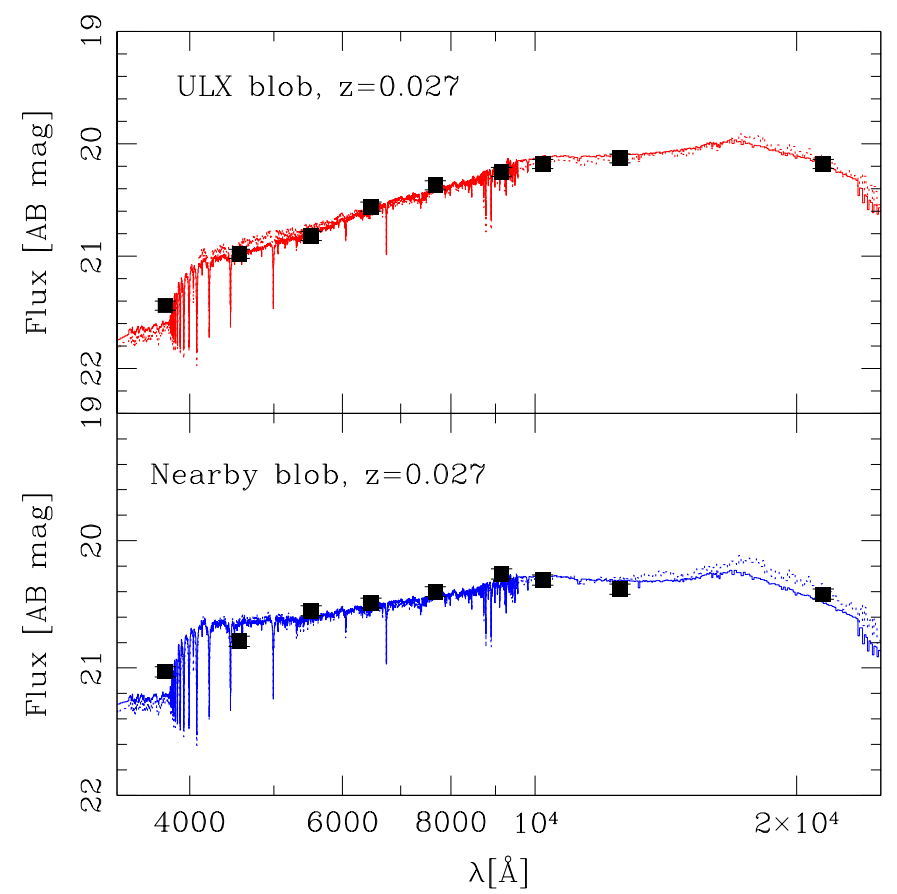

Fig. 4. Spectral energy distribution (SED) of the ULX (upper section) and nearby (lower section) blobs from broadband photometric data (black squares) and their best fit population synthesis models (thin lines). In both panels, the dotted line shows the fit with solar metallicity value $Z_{\odot}$, while the solid line shows the fit with metallicity $0.4 Z_{\odot}$. The contribution from major emission lines was subtracted from the photometric data. Error bars are about of the size of the symbols.

using the best fit model described above. This correction increased the measured line fluxes by at most $20 \%$ for the $\mathrm{H} \beta$ line. Finally, emission line fluxes were measured by Gaussian fits via the splot routine of IRAF as well as our own software. Table 3 shows the line luminosities computed for each emission line present in the spectra. The $90 \%$ confidence statistical errors or 95\% upper limits are also indicated. Owing to our limited resolution, $\mathrm{H} \alpha$ and $[\mathrm{NII}] \lambda \lambda 6548,6583$ are blended. In either case, the flux of the single Gaussian fit is dominated by the $\mathrm{H} \alpha$ side and leaves some excess residuals longward of $\mathrm{H} \alpha$ around [NII]. Forcing a double Gaussian fit gives a small flux for [NII]. A rough estimation of the $[\mathrm{NII}] / \mathrm{H} \alpha$ ratio gives a value of $\sim 0.1$. However, the line profiles are not necessarily exact Gaussian and a slight deviation from Gaussian may cause a large flux misestimation of the subdominant [NII] line. Therefore, the results of a single Gaussian fit to the $\mathrm{H} \alpha+[\mathrm{NII}]$ feature are shown in Table 3 and we use them as representative values for $\mathrm{H} \alpha$. Thus the $\mathrm{H} \alpha$ luminosities shown in Table 3 are subject to contaminations from [NII]. Instead, for the R500B spectrum of the nearby blob, we were able to obtain a reasonable multicomponent fit with a model including $\mathrm{H} \alpha$ and [NII] $\lambda \lambda 6548,6583$, where the flux ratio between [NII] $\lambda 6548$ and $\lambda 6583$ lines is fixed to the theoretical value of 3.0. For this particular case, 
Table 3. Emission line luminosities $\left[10^{39} h_{70}^{-2} \mathrm{erg} \mathrm{s}^{-1}\right]$ (90\% statistical errors or $95 \%$ upper limits).

\begin{tabular}{ccccccccc}
\hline \hline Grism & Object & $L([\mathrm{OII}])$ & $L(\mathrm{H} \beta)$ & $L\left([\mathrm{OIII}]^{1}\right)$ & $L\left([\mathrm{OIII}]^{2}\right)$ & $L([\mathrm{OI}])$ & $L(\mathrm{H} \alpha+[\mathrm{NII}])$ & $L([\mathrm{SII}])$ \\
\hline \multirow{2}{*}{ R500B } & ULX blob & $2.18 \pm 0.03$ & $1.16 \pm 0.05$ & $1.34 \pm 0.02$ & $4.15 \pm 0.05$ & $\leq 0.16$ & $4.56 \pm 0.06$ & $0.70 \pm 0.07$ \\
& Nearby blob & $6.34 \pm 0.32$ & $2.18 \pm 0.24$ & $0.55 \pm 0.11$ & $1.62 \pm 0.08$ & $\leq 0.21$ & $4.53 \pm 0.09$ & $1.59 \pm 0.12$ \\
\hline \multirow{2}{*}{ R500R } & ULX blob & - & $1.67 \pm 0.06$ & $2.03 \pm 0.06$ & $5.58 \pm 0.06$ & $0.22 \pm 0.04$ & $5.30 \pm 0.05$ & $1.13 \pm 0.04$ \\
& Nearby blob & - & $2.10 \pm 0.12$ & $1.5 \pm 0.10$ & $3.35 \pm 0.06$ & $\leq 0.13$ & $10.56 \pm 0.10$ & $1.87 \pm 0.07$ \\
\hline
\end{tabular}

Notes. ${ }^{(1)}[\mathrm{OIII}] \lambda 4959,{ }^{(2)}[\mathrm{OIII}] \lambda 5007$.
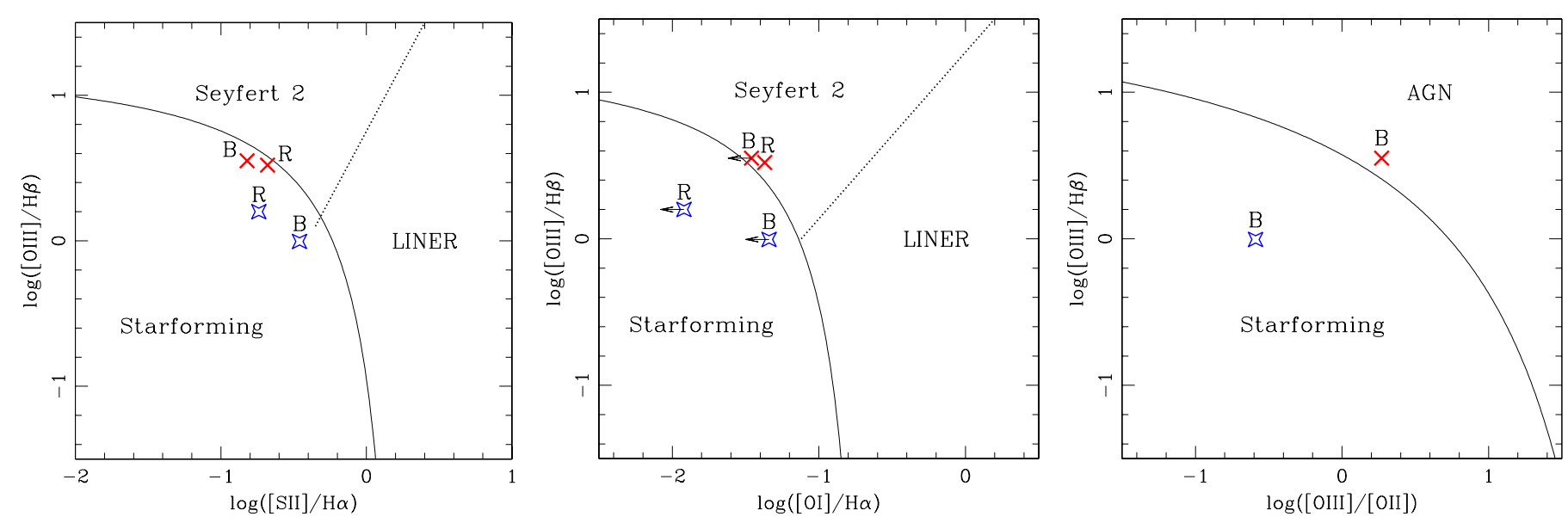

Fig. 5. Diagnostic $\log ([\mathrm{OIII}] / \mathrm{H} \beta)$ vs. $\log ([\mathrm{SII}] / \mathrm{H} \alpha)($ left $), \log ([\mathrm{OIII}] / \mathrm{H} \beta)$ vs. $\log ([\mathrm{OI}] / \mathrm{H} \alpha)($ middle $), \operatorname{and} \log ([\mathrm{OIII}] / \mathrm{H} \beta)$ vs. $\log ([\mathrm{OIII}] /[\mathrm{OII}])($ right $)$ diagrams. In the left and middle diagrams, the boundary lines were taken from Kewley et al. (2006), while in the right diagram the boundary line was taken from Evans \& Dopita (1985). The ULX blob is represented with a red cross, while the nearby blob is shown with a blue star. The B symbol indicates the line ratios computed from R500B grism, while the R shows the line ratios computed from R500R grism. Statistical errors are smaller than the symbol sizes, except those for $\log ([\mathrm{OI}] / \mathrm{H} \alpha)$.

we obtain $L(\mathrm{H} \alpha)=(4.46 \pm 0.05) \times 10^{39}$ and $L([\mathrm{NII}] \lambda 6583)=$ $(1.04 \pm 0.05) \times 10^{38} h_{70}^{-2} \mathrm{erg} \mathrm{s}^{-1}$. We expect that systematic errors, such as a slight differences of seeing, slit positions, and background sky subtraction, are larger and might have caused the differences between the same lines observed with R500B and R500R gratings.

In order to see if the emission lines of the ULX nebula are solely ionized by OB stars or there is significant contribution from another source, we plot line ratios on two diagnostic diagrams that are customarily used to classify narrow emission line galaxies into Seyferts, LINERs, and HII galaxies. We are unable to use the most common diagram with $\log ([\mathrm{OIII}] / \mathrm{H} \beta)$ versus $\log ([\mathrm{NII}] / \mathrm{H} \alpha)$ because of the blending problem. However, Fig. 5 (left, middle, and right panels) shows the Kewley et al. (2006) $\log ([\mathrm{OIII}] / \mathrm{H} \beta)$ versus $\log ([\mathrm{SII}] / \mathrm{H} \alpha)$ (left), $\log ([\mathrm{OIII}] / \mathrm{H} \beta$ ) versus $\log ([\mathrm{OI}] / \mathrm{H} \alpha$ ) (middle), and the Evans \& Dopita $(1985) \log ([\mathrm{OIII}] / \mathrm{H} \beta)$ versus $\log ([\mathrm{OIII}] /[\mathrm{OII}])$ (right) star-forming-AGN diagnostic diagrams for our ULX blob. It can be seen that the ULX blob (red crosses) is located on the borderlines between star formation and Seyfert/AGN regimes in all diagrams. Only a few percent of HII/star-forming galaxies without AGNs from Veilleux \& Osterbrock (1987) data fall into the upper right quadrant with respect to the ULX blob position. On the other hand, the nearby blob (blue star) shows a line ratio typical of a star-forming regions in both diagnostic diagrams. We estimated an upper limit value for the [OI] luminosity of the nearby blob, according to the limit flux measurable in their spectra. The [OI] upper limits for the nearby blob are also plotted in Fig. 5 and listed in Table 3. Thus, the presented results suggest a noticeable contribution from accretion process to the ionizing radiation, producing the emission line nebula in the ULX blob.

\section{Discussion}

The star formation rate and stellar masses of the ULX and the nearby blobs are very similar. The star formation rates inferred by the SED fits $\left(\sim 0.02-0.16 M_{\odot} \mathrm{yr}^{-1}\right)$ are close to those estimated by their $\mathrm{H} \alpha$ and [OII] line luminosities ( $\sim 0.02-0.08 M_{\odot} \mathrm{yr}^{-1}$ ) using the Kennicutt et al. (2009) formulas. The ULX thus resides in an actively star-forming region. Our slit size of $1.2^{\prime \prime}$ corresponds to $\sim 650 h_{70}^{-1} \mathrm{pc}\left(D \approx 110 h_{70}^{-1} \mathrm{Mpc}\right)$, while the emission line regions of nearby ULX nebulas typically extend to $\$ 500$ pc (Pakull et al. 2006; Abolmasov et al. 2007; hereafter A07). The stellar masses derived from the SED fits, $\sim(1.2-3.6) \times 10^{7} M_{\odot}$, are much higher than typical stellar masses of OB associations $\left(10^{2}-10^{4} M_{\odot}\right.$; Fall \& Chandar 2012). However, it has been inferred that star clusters with higher stellar mass values exist in the Galaxy $\left(\sim 10^{5} M_{\odot}\right.$; Rahman et al. 2011) and other external galaxies $\left(\sim 10^{6} M_{\odot}\right.$; Larsen 2009 ; Weidner et al. 2010). Then, the stellar mass estimated for the blobs might correspond with a massive star cluster composed of a group of OB associations. Thus, we would expect that the emission line spectra of the ULX blob in our observations consist of a mixture of HII regions ionized by OB stars and a nebula ionized by the ULX.

In order to see if it is reasonable to expect the contribution from accretion, we compare the [OIII] $\lambda 5007$ to X-ray $(0.3-10 \mathrm{keV})$ luminosity ratio of the ULX blob with those of known ULX nebulas. The $L_{\lambda 5007} / L_{0.3-10 \mathrm{keV}} \approx 0.15$ for our ULX blob is comparable to those of two of the ULX nebulas studied by A07, namely NGC 6946 X-1 and NGC 7331 X-1, with $L_{\lambda 5007} / L_{0.3-10 \mathrm{keV}} \approx 0.1$ and 0.06 , respectively $(0.3-10 \mathrm{keV}$ luminosities are from Swartz et al. 2011). Furthermore, even with a low value of $[\mathrm{NII}] / \mathrm{H} \alpha \sim 0.1$ for our ULX blob, which was 
obtained with an unreliable fit in Sect. 4, the ULX contribution is still pausible. In fact, the ULX nebula associated with NGC 5204 $\mathrm{X}-1$ by $\mathrm{A} 07$ has a high value of $[\mathrm{OIII}] / \mathrm{H} \beta \sim 5$ and still has a rather low value of $[\mathrm{NII}] / \mathrm{H} \alpha \sim 0.13$. Low-mass/low-metallicity photoionized or shock-heated nebulae are expected to show low values of the $[\mathrm{NII}] / \mathrm{H} \alpha$ ratio, while keeping the $[\mathrm{OIII}] / \mathrm{H} \beta$ ratio relatively unchanged (Groves et al. 2006; Dopita et al. 2012). Thus, we may reasonably expect significant contributions of the ULX accretion to the line ratios, which pushes the ULX nebula toward the Seyfert regime in the emission line diagnostic diagrams. In addition, as mentioned above, because of the low stellar mass estimated for our ULX nebula and the low [NII]/H $\alpha$ ratio, we prefer the subsolar metallicity value used in the SED fits with its derived properties as the most likely value (Table 2).

It is not surprising that we did not detect HeII $\lambda 4686$ emission, which is common in nearby ULXs. Our line detection upper limit is $L_{\mathrm{lim}} \sim 3 \times 10^{38} h_{70}^{-2} \mathrm{erg} \mathrm{s}^{-2}$, while the range of the HeII line luminosities for the nearby nebulae is $L_{\mathrm{HeII}} \sim(1-11) \times$ $10^{36} h_{70}^{-2} \operatorname{erg~s}^{-2}$ (A07).

While our observational clues about the nature of the ULX, especially in terms of the constrains on the mass of the black hole of the ULX, are limited, it might be instructive to present a few possible cases that are consistent with our observations and to discuss their implications. The ionization of the ULX nebula may be either photoionization or heating by a shock with velocity $V \gtrsim 100 \mathrm{~km} \mathrm{~s}^{-1}$ (e.g., Allen et al. 2008; Dopita et al. 2012). Assuming a photoionization scenario, let $f$ be the fraction of $\mathrm{H} \beta$ emission that is contributed by the ULX accretion. Naturally, the value of $f$ is essentially unconstrained. Since the SFR and associated $\mathrm{H} \alpha$ and $\mathrm{H} \beta$ fluxes from the SED fits vary by a factor of several as we vary the assumed metalicity, a reasonable estimate of $f$ can not be obtained by comparing the Balmer fluxes expected from the SFR from the population synthesis results and from the ULX blob. Nevertheless, the $L_{[\mathrm{OIII}]} / L_{\mathrm{H} \beta}$ values of nearby ULX nebulae range from $\sim 2-7$ (A07). For example, $L_{[\mathrm{OIII}]} / L_{\mathrm{H} \beta} \sim 7$ for the case with high ULX ionization (from NGC 6946 X-1 observation) and $L_{[\mathrm{OIII}]} / L_{\mathrm{H} \beta} \sim 2$ (from our nearby nebula) for the case with $\mathrm{OB}$ star ionization. Then, we obtain $f \sim 0.35$ to have $L_{[\mathrm{OIII}]} / L_{\mathrm{H} \beta} \sim 3.5$, which is the observed value for our ULX blob. Instead, if we assume $L_{[\mathrm{OIII}]} / L_{\mathrm{H} \beta} \sim 4$ for our ULX nebula, $f \sim 0.75$. Here we discuss the implications in the following cases of accretion.

a) The ULX accretion is Eddington-limited (e.g., the case of a standard $\alpha$-disk; Shakura \& Sunyaev 1973) and the ULX nebula is shock-heated. In this case, we can assume the bolometric luminosity is dominated by the $\mathrm{X}$-ray emission. Then, $L_{\mathrm{bol}} \sim 2 \times 10^{40} \mathrm{erg} \mathrm{s}^{-1}$, and the black hole mass (BH) would be $M_{\mathrm{BH}}>140 M_{\odot}$

b) The ULX accretion is Eddington-limited and the ULX nebula is photoionized. Under this scenario, Eq. (5) of A07 implies an ionizing UV radiation from ULX accretion disk with $L_{\mathrm{UV}} \sim 1.2 \times 10^{41} f h_{70}^{-2} \mathrm{erg} \mathrm{s}^{-1}$. We also have to consider the ionizing UV luminosity in addition to the X-ray luminosity, and therefore the bolometric luminosity would become $L_{\text {bol }} \sim(2+12 f) \times 10^{40} \mathrm{erg} \mathrm{s}^{-1}$. The corresponding BH mass would be $M_{\mathrm{BH}}>140(1+6 f) M_{\odot}$ in order not to violate the Eddington-limit. If $f=0.5$, the $\mathrm{BH}$ mass would be $M_{\mathrm{BH}} \gtrsim$ $1100 M_{\odot}$.

c) The ULX accretion is a super critical accretion disk (SCAD) and the ULX nebula is shock-heated. The SCAD is either the case in which most of the accreted energy is advected onto the black hole (slim disk; Watarai et al. 2001) or in which a bulk of energy output is transferred to outflows
(Poutanen et al. 2007). Under SCAD models a radiation output (true luminosity) can exceed the Eddington limit by a factor 2-3. A further boosting of a factor of a few is possible for an apparent luminosity if it is combined with a mild collimation (Feng \& Soria 2011, and references therein). Thus, in the SCAD model with a shock heating of the ULX nebula, the apparent luminosity can be $\sim 10$ times higher than the corresponding Eddington limit. Since the observed X-ray luminosity is expected to be the bolometric luminosity, the $\mathrm{BH}$ mass can be as low as $M_{\mathrm{BH}} \gtrsim 15 M_{\odot}$, which is consistent with a stellar mass $\mathrm{BH}$.

d) The ULX accretion is a SCAD and the ULX nebula is photoionized. In this case, we should treat the X-ray and UV luminosities differently. The mild collimation boost only applies to the apparent X-ray luminosity, but does not apply to the UV luminosity, which is implied by the $\mathrm{H} \beta$ emission. If the SCAD allows the emission of $b_{\mathrm{S}}$ times more radiation than the Eddington luminosity and the collimation boosting factor is $b_{\mathrm{C}}$, the implied $\mathrm{BH}$ mass becomes $M_{\mathrm{BH}} \gtrsim$ $140\left(1 / b_{\mathrm{C}}+12 f\right) / b_{\mathrm{S}} M_{\odot}$. If we assume $b_{\mathrm{S}} \approx b_{\mathbf{C}} \approx 3$ and $f=$ 0.5 , then $M_{\mathrm{BH}} \gtrsim 300 M_{\odot}$.

Under the scenarios (b) and (d), the existence of an IMBH $\left(M_{\mathrm{BH}} \sim 10^{2}-10^{4} M_{\odot}\right)$ is implied. A major problem with the IMBH scenario is that there is no known black hole in this mass range, even though it has been theoretically suggested that the IMBHs can form from collapsed massive population III stars or at the nucleus of star clusters (Feng \& Soria 2011, and references therein). On the other hand, for $f \sim 0.1$ under the scenario (d), the inferred $\mathrm{BH}$ mass is $M_{\mathrm{BH}} \gtrsim 70 M_{\odot}$. There is at least one known example of a black hole with a similar mass, which is the remnant of the black hole merger gravitational wave source GW150914 $\left(M_{\mathrm{BH}}=62 \pm 4 M_{\odot}\right.$; Abbott et al. 2016). Finally, the existence of a couple of ULXs powered by an accreting neutron star should also be mentioned. In this scenario, a neutron star reaches a $L_{\mathrm{X}}>10^{40} h_{70}^{-2} \mathrm{erg} \mathrm{s}^{-1}$ accreting at superEddington rates in a binary system (M82 X2, Bachetti et al. 2014; NGC 7793 P13, Fürst et al. 2016, Israel et al. 2017).

\section{Conclusions}

We have discovered a new ULX at $z=0.027$ with Chandra observations in the AKARI NEP Deep Field. The ULX, ANEPDCXO104 is located $\sim 3.1 \mathrm{kpc}$ away from the center of the galaxy NEP J175536.7+664546.8, and it is associated with a high excitation emission line nebula. The location of the ULX coincides with one of several HII region blobs along spirals of the galaxy.

The X-ray spectrum shows a power-law shape with slope $\Gamma=$ $2.2 \pm 0.4$ without evidence for intrinsic absorption. The X-ray luminosity estimated for our ULX is $L_{0.5-7 \mathrm{keV}} \approx 2.4 \pm 0.5 \times$ $10^{40} h_{70}^{-2} \operatorname{erg~s}^{-1}$.

The optical spectrum of the ULX blob shows emission lines typical of active regions with strong Balmer, [OII], and [OIII] emission lines. The [OI] $\lambda 6300$ and HeI $\lambda 6876$ lines are also detected. The spectrum of other HII blob nearby was also taken within the same slit, presenting the same redshift and similar spectral features.

We applied a SED fitting to the photometric data. The fits for both blobs revealed very similar properties, which are well represented by a continuous, almost constant SFR $\left(\sim 0.02-0.16 M_{\odot} \mathrm{yr}^{-1}\right)$ that started $0.09-2$ Gyr ago. These SFR values are close to those estimated by their $\mathrm{H} \alpha$ and [OII] luminosities $\left(\sim 0.02-0.08 M_{\odot} \mathrm{yr}^{-1}\right)$. The stellar masses estimated for both blobs $\left(\sim 1.2-3.6 \times 10^{7} M_{\odot}\right)$ are much higher than typical 
values of OB associations $\left(10^{2}-10^{4} M_{\odot}\right.$; Fall \& Chandar 2012) and they might correspond with massive star clusters composed of a group of $\mathrm{OB}$ associations.

In order to see if the emission lines of the ULX nebula are produced solely by OB stars or by contributions from other sources, we used three diagnostic diagrams: the $\log ([\mathrm{OIII}] / \mathrm{H} \beta)$ versus $\log ([\mathrm{SII}] / \mathrm{H} \alpha), \log ([\mathrm{OIII}] / \mathrm{H} \beta)$ versus $\log ([\mathrm{OI}] / \mathrm{H} \beta)$ diagrams (from Kewley et al. 2006), and the $\log ([\mathrm{OIII}] / \mathrm{H} \beta$ ) versus $\log ([\mathrm{OIII}] /[\mathrm{OII}])$ diagram (from Evans \& Dopita 1985). The ULX blob is located on the borderlines between star formation and Seyfert/AGN regimes in all diagrams. These results are in contrast with those of the nearby blob, which occupies the HII regimes.

The ratio between [OIII] $\lambda 5007$ and X-ray $0.3-10 \mathrm{keV}$ luminosities is $L_{\lambda 5007} / L_{0.3-10 \mathrm{keV}} \approx 0.15$ for our ULX blob; this is a comparable value to those of other ULX nebulae (Abolmasov et al. 2007). Thus, based on our results we may reasonably expect significant contributions of the ULX accretion to the emission lines, moving the location of the ULX nebula toward the Seyfert regime in the diagnostic diagrams.

Owing to the distance to our ULX, the analyses are limited by the spatial resolution. High spatial resolution spectroscopy, such as that available with the Space Telescope Imaging Spectrograph (STIS) attached to the Hubble Space Telescope (HST), would give a superb emission line diagnostics of this ULX with much less contamination from the surrounding star-forming regions.

Acknowledgements. This research was partially supported by Consejo Naciona de Ciencia y Tecnología (CONACyT, México; grants 179662 and 252531) and the Dirección General de Asuntos del Personal Académico (DGAPA-UNAM, México) through the PAPIIT program (IN104216). J.D. thanks to DGAPA for the fellowships awarded to do this research. T.I., H.H., and Y.U. are thankfu for the financial support by the Grant-in-Aid for Scientific Research (26400216 $21340042,24650145,26400228$ ) from Japan Society for the Promotion of Science (JSPS). M.K. acknowledges financial support by DFG grant KR3338/3-1. This study is based on data principally collected using the Gran Telescopio de Canarias, operated by the Instituto de Astrofísica de Canarias, under active support of the Spanish Government and the Local Government from the Canary Islands through the European Funds for the Regional Development (FEDER) provided by the European Union, including participation of the Instituto de Astronomía de la Universidad Nacional Autónoma de México (IA-UNAM), the Instituto Nacional de Astrofísica, Óptica y Electrónica (INAOE, México), and the University of Florida (United States); the Chandra X-ray Observatory, operated by the Smithsonian Astrophysical Observatory for and on behalf of the Nationa Aeronautic Space Administration (NASA); the Subaru telescope operated by the National Astronomical Observatory of Japan (NAOJ) and the Canada-FranceHawaii Telescope (CFHT) operated by the National Research Council (NRC) of
Canada, the Institut National des Sciences de l'Univers of the Centre National de la Recherche Scientifique of France, and the University of Hawaii.

\section{References}

Abbott, B., Abbott, R., Abbott, T., et al. 2016, ApJ, 818, L22 Abolmasov, P., Fabrika, S., Sholukhova, O., et al. 2007, Astrophys. Bull., 62, 36 Allen, C. 1976, Astrophysical Quantities (London: Athlone Press), 264

Allen, M., Groves, B., Dopita, M., et al. 2008, ApJS, 178, 20

Bachetti, M., Harrison, F., Walton, D., et al. 2014, Nature, 514, 202

Begelman, M. 2002, ApJ, 568, 97

Bolzonella, M., Miralles, J., \& Pelló, R. 2000, A\&A, 363, 476

Bruzual, G., \& Charlot, S. 2003, MNRAS, 344, 1000

Colbert, E., \& Mushotzky, R. 1999, ApJ, 519, 89

Dopita, M., Payne, J., Filipovic, M., et al. 2012, MNRAS, 427, 956

Evans, I., \& Dopita, M. 1985, ApJS, 58, 125

Fabrika, S., Ueda, Y., Vinokurov, A., Sholukhova, O., \& Shidatsu, M. 2015, Nature Phys., 11, 551

Fall, S., \& Chandar, R. 2012, ApJ, 752, 96

Feng, H., \& Soria, R. 2011, New Astron. Rev., 55, 166

Fürst, F., Walton, D., Harrison, F., et al. 2016, ApJ, 831, 14

Goad, M., Roberts, T., Knigge, C., et al. 2002, MNRAS, 335, 67

Groves, B., Heckman, T., \& Kauffmann, G. 2006, MNRAS, 371, 1159

Gutiérrez, C., \& Moon, D. 2014, ApJ, 797, 7

Hanami, H., Ishigaki, T., Fujishiro, N., et al. 2012, PASJ, 64, 70

Israel, G., Papitto, A., Esposito, P., et al. 2017, MNRAS, 466, 48

Kaaret, P., Ward, M., \& Zezas, A. 2004, MNRAS, 351, 83

Kalberla, P., Burton, W., Hartmann, D., et al. 2005, A\&A, 440, 775

Kennicutt, R., Hao, C., Calzetti, D., et al. 2009, ApJ, 703, 1672

Kewley, L., Grobes, B., Kauffmann, G., et al. 2006, MNRAS, 372, 961

King, A., Davies, M., Ward, M., et al. 2001, ApJ, 552, 109

Krumpe, M., Miyaji, T., Brunner, H., et al. 2015, MNRAS, 446, 911

Kuntz, K., Gruendi, R., Chu, Y., et al. 2005, ApJ, 620, 31

Larsen, S. 2009, A\&A, 494, 539

Lehmann, I., Becker, T., Fabrika, S., et al. 2005, A\&A, 431, 847

Liu, J., Bregman, J., \& Seitzer, P. 2004, ApJ, 602, 249

Makishima, K., Kubota, A., Mizuno, T., et al. 2000, ApJ, 535, 632

Matsuhara, H., Wada, T., Matsuura, S., et al. 2006, PASJ, 58, 673

Murata, K., Matsuhara, H., Wada, T., et al. 2013, A\&A, 559, A132

Oi, N., Matsuhara, H., Murata, K., et al. 2014, A\&A, 566, A60

Pakull, M., \& Mirioni, L. 2002, ArXiv e-prints [arXiv: astro-ph/0202488]

Pakull, M., Grisé, F., \& Motch, C. 2006, IAUS, 230, 293

Poutanen, J., Lipunova, G., Fabrika, S., et al. 2007, MNRAS, 377, 1187

Rahman, M., Moon, D., \& Matzner, C. 2011, ApJ, 743, L28

Salpeter, E. 1955, ApJ, 121, 161

Shakura, N., \& Sunyaev, R. 1973, A\&A, 24, 337

Shim, H., Im, M., Ko, J., et al. 2013, ApJS, 207, 37

Swartz, D., Soria, R., Tennant, A., et al. 2011, ApJ, 741, 49

Takagi, T., Matsuhara, H., Wada, T., et al. 2007, PASJ, 59, S557

Veilleux, S., \& Osterbrock, D. 1987, ApJ, 63, 295

Watarai, K., Mizuno, T., \& Mineshige, S. 2001, ApJ, 549, L77

Weidner, C., Bonnell, I., \& Zinnecker, H. 2010, ApJ, 724, 1503

Zezas, A., Fabbiano, G., Rots, A., et al. 2002, ApJ, 577, 710 